\title{
Interface size dependence of the Josephson critical behaviour in pyrolytic graphite TEM lamellae
}

\author{
Ana Ballestar, ${ }^{1}$ Tero T. Heikkilä, ${ }^{2}$ and Pablo Esquinazi ${ }^{1,}$ * \\ ${ }^{1}$ Division of Superconductivity and Magnetism, Institut für Experimentelle Physik II, \\ Universität Leipzig, Linnéstraße 5, D-04103 Leipzig, Germany \\ ${ }^{2}$ Department of Physics and Nanoscience Center, University of Jyväskylä, \\ P.O. Box 35 (YFL), FI-40014 University of Jyväskylä, Finland
}

\begin{abstract}
We have studied the transport properties of TEM lamellae obtained from a pyrolytic graphite sample with electrical contacts at the edges of the embedded interfaces. The temperature dependence of the resistance as well as the current-voltage characteristic curves are compatible with the existence of Josephson coupled superconducting regions. The transition temperature at which the Josephson behavior sets in, decreases with the interface width and vanishes for width below $200 \mathrm{~nm}$. This unexpected behavior is apparently due to the influence of weak localization effects on the superconducting critical temperature.
\end{abstract}

The properties of surfaces and interfaces in solids can be fundamentally different from those of the corresponding bulk material. Differences in the carrier dispersion relation compared to the quadratic free electron case can strongly affect the electronic properties of the interfaces. Conductivity experiments are extremely sensitive to such effect, specially in samples where superconductivity at certain interfaces compete with the rest of the nonsuperconducting matrix 1, 2. The possibility of high-temperature superconductivity at surfaces and interfaces has attracted the attention of the physics community since the earliest 60's [3. Superconductivity has been found at the interfaces between oxide insulators [1] and between metallic and insulating copper oxides with critical temperature $T_{c} \gtrsim 50 \mathrm{~K} 2$. Interfaces in pure [4-7] as well as doped Bi bicrystals can show superconductivity up to $T_{c} \simeq 21 \mathrm{~K}[8$, although $\mathrm{Bi}$ as bulk is not a superconductor.

High-temperature superconductivity has been predicted to occur at topologically protected flat bands on the surface [9] or at certain rhombohedral-Bernal interfaces [10, 11] in graphite [12. Inclusions of rhombohedral graphite ordered regions have been found embedded in bulk Bernal graphite [13] as well as in exfoliated multilayer graphene films 14, both taken from highly oriented pyrolytic graphite (HOPG) samples. The possible existence of high-temperature superconductivity embedded in disordered graphite has already been speculated 40 years ago [15, 16. Since then different studies were published in graphite [17] as well as in doped disordered carbon samples 18 providing indications of high transition temperatures. The existence of quasi two-dimensional (2D) interfaces in HOPG and in Kish graphite samples has been known long time ago [19], but their extraordinary properties were reported only recently as a result of contacting the edges of the embedded interfaces found in transmission electron micro-

*Electronic address: esquin@physik.uni-leipzig.de scope (TEM) graphite lamellae [20]. Evidence for granular superconductivity in graphite flakes has been independently observed in the field hysteresis and field periodic oscillations in high resolution magnetoresistance measurements as well as in SQUID magnetization measurements of HOPG samples with interfaces, for a review see [17.

Sample lateral-size dependent effects in the magnetoresistance of graphite have been already reported and explained in terms of the large (several microns) mean free path of the carriers within the graphene layers [21, 22. The phenomenon we describe in this letter, however, has not been reported yet for graphite and it is related to the transition temperature $T_{c}$ characteristic of the Josephson effect found at graphite interfaces [20]. We have found that this $T_{c}$ decreases with the width of the $2 \mathrm{D}$ interface region. The observed behavior may clarify the origin of certain differences in the temperature dependence of the resistance, especially the temperature of the maximum $T_{\max }$ in the resistance of bulk graphite samples and of small graphite flakes with interfaces. We propose that, at least part of this difference is related to the area of the embedded interfaces where superconductivity exists. We expect that there exists a direct correspondence between the true superconducting critical temperature and the temperature $T_{c}$ (at which the Josephson behavior starts to be measurable) and that $T_{\max } \sim T_{c}$.

TEM lamellae, with thickness between $80 \mathrm{~nm}$ and $800 \mathrm{~nm}$, were cut with the $\mathrm{Ga}^{+}$beam of a dual beam microscope (FEI Nanolab XT 200) from the same bulk HOPG sample grade ZYA (0.4 $4^{\circ}$ rocking curve width). We previously covered the sample surface with a wolframcarbide (WC) film, deposited using Electron Beam Induced Deposition (EBID), to avoid the penetration of the $\mathrm{Ga}^{+}$ions in the graphite structure of the lamella 20. The lamella thickness corresponds to the width of the graphene planes and therefore to the width of the internal interfaces, as they run parallel to the graphene planes, see TEM images in [19, 20]. Moreover, electron backscattering diffraction (EBSD) measurements showed the existence of grains of several micrometers in the $a b$ - 


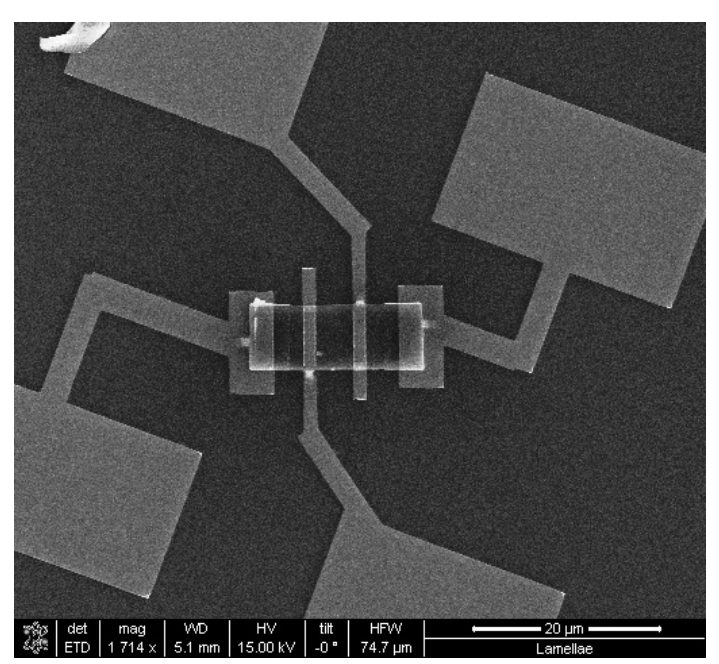

FIG. 1: Scanning electron microscope image of one of the studied lamella (thickness $200 \mathrm{~nm}$ ). The brighter areas correspond to the four $\mathrm{Pt} / \mathrm{Au}$ electrodes placed in line as usual in the four probes method. Note that the outer contacts (used for the input current) are placed at the edges of the sample, i.e. at the edges of the interfaces. The inner ones are used to measure the corresponding response (voltage) coming from these interfaces. The $c$-axis of the graphite structure (normal to the graphene planes) is parallel to the substrate, normal to the input current direction.

plane [19, 21]. The transport measurements were done using a four-probe electrode technique, in the conventional configuration, see Fig. 1.

Figure 2 shows the temperature dependence of the voltage for four TEM lamellae measured at a constant input current of $1 \mathrm{nA}$. For clarity and taking into account the differences in the absolute resistance between the samples, the voltages of three lamellae are normalised at their maxima (left $y$-axis). The absolute resistance of a bulk HOPG samples as well as the voltage of a fourth lamella are shown in the same figure (right $y$-axis). Note that the measurements of the four lamellae presented in Fig. 2 were done using $1 \mathrm{nA}$ current because the observed transitions shift to lower temperatures the larger the input current, as expected in a system with superconducting grains.

In Fig. 2 we recognize clear drops of the voltage at different temperatures for lamellae with thickness $d \gtrsim$ $200 \mathrm{~nm}$, while for a thinner lamella $(d=100 \mathrm{~nm})$ a semiconducting-like behavior is observed down to $2 \mathrm{~K}$, the lowest measured temperature. A similar semiconducting like dependence is measured for bulk graphite or multilayer graphene samples without interfaces, independently of the lateral size of the sample. For lamellae with thickness $d \gtrsim 200 \mathrm{~nm}$ the measured voltage at low enough temperatures and currents fluctuates around zero within $\lesssim| \pm 20| \mathrm{nV}$. The finite sensitivity of the electronics and the noise do not allow, strictly speaking, to observe a zero

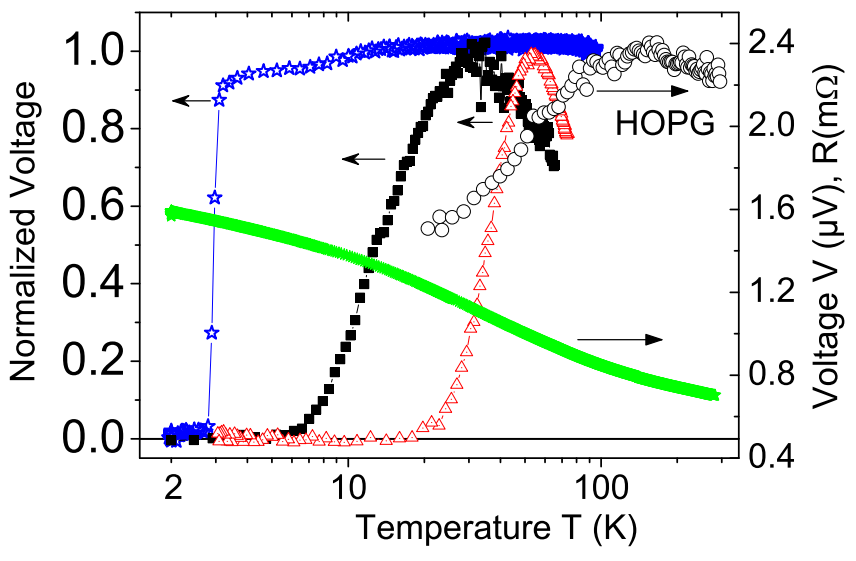

FIG. 2: Left $y$-axis: normalized voltage measured at $1 \mathrm{nA}$ current vs. temperature of three lamellae with different thickness, namely 200,300 and $500 \mathrm{~nm}( \pm 20 \mathrm{~nm})$ for $(\star, \mathbf{\square}, \triangle)$ and different normalization voltages, respectively. Right $y$-axis: Green symbols $(*)$ show the absolute voltage vs. temperature at $1 \mathrm{nA}$ measured for a lamella with thickness $100 \pm 10 \mathrm{~nm}$. (o): Resistance $R$ vs. temperature measured for the original bulk HOPG sample (current $1 \mu \mathrm{A}$ ) from where the lamellae were obtained.

resistance state below $T_{c}$. For that purpose one needs to show the existence of persistent currents, as done in 23, for example. The current-voltage $(I-V)$ characteristic curves obtained for the studied lamellae are consistent with the existence of Josephson junctions, as Fig. 3 shows for an $800 \mathrm{~nm}$ thick lamella at different temperatures.

Thermal fluctuation effects dominate in a Josephson junction when the thermal energy $k_{B} T$ is larger than the Josephson coupling energy $E_{J}=(\hbar / 2 e) I_{c}$ ( $I_{c}$ is the critical Josephson current). Early theoretical work provides an adequate framework to understand the measured $I-V$ curves including the influence of thermal fluctuations on Josephson junctions [24, 25]. It is found that there is always a finite resistance, even below $I_{c}$, due to thermally activated phase processes. Using the differential equation proposed in 24, we fit the measured $I-V$ curves with the critical current $I_{c}(T)$ as the only free parameter. To present the results in actual units of current and voltage the values of the resistance in the normal state $R_{n}$ have been used, obtained from the slopes of the $I-V$ curves well above $I_{c}(T)$, once the linear regime was reached. From the fits to the $I-V$ curves shown in Fig. 3 we obtained $I_{c}(\mu \mathrm{A})=0.162$, $0.158,0.091$, and 0.013 at $T(\mathrm{~K})=40,50,70,85 \mathrm{~K}$, respectively. The rather unusual behavior of the $I-V$ curves at large voltages, see Fig. 3, is due to the transition from the metallic- to a semiconducting-like temperature dependence at large currents, similar to the magnetic-field driven metal-insulator transition (MIT) in HOPG with interfaces [26. 


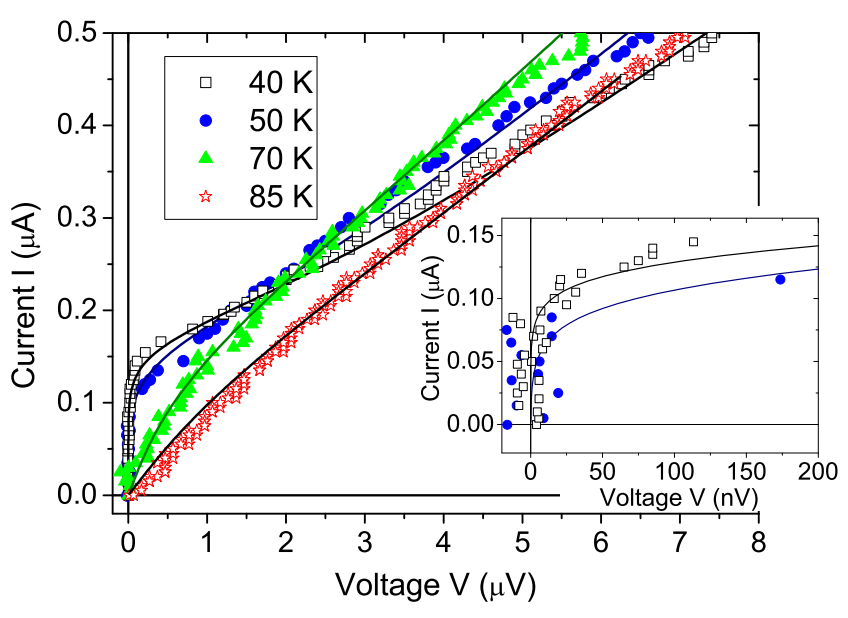

FIG. 3: Current vs. voltage characteristic curves at different temperatures for one of the studied lamellae (thickness $d=$ $800 \mathrm{~nm}$ ). The inset blows out the data at low currents and low voltages. The continuous lines were calculated following [24] with the critical Josephson current as the only free parameter, see text.

In Fig. 2 (right $y$-axis) we show the temperature dependence of the resistance of a bulk piece of HOPG ZYA sample, a part of the same bulk piece from where all the lamellae here studied were obtained. The measured resistance shows a maximum at $T \simeq 150 \mathrm{~K}$. This maximum is not universal but depends on the internal structure of the graphite sample. Our hypothesis is that the position of the maximum in the temperature dependence of the resistance is directly related to the existence of interfaces and their properties. Following transport results in graphite flakes [17 and an interpretation of the magnetic-field driven MIT in graphite bulk samples [26, we argue that the maximum in the electrical resistance indicates the temperature below which a Josephson-like coupling between the superconducting regions within the interfaces starts to be measurable. In this case one possible reason for the change in the temperature dependence of the resistance could be the size of the superconducting regions or the superconducting/normal ratio.

Figure 4(a) shows $T_{c}$ obtained at $1 \mathrm{nA}$ vs. the lamella thickness $d$. We observe that the defined $T_{c}$ decreases nearly linearly with decreasing $d$ and extrapolates to zero at a finite thickness $d \sim 160 \mathrm{~nm}$. To check whether this decrease of $T_{c}$ is not due to an increase in the current density related to the change in the lamellae geometry, we have measured also the current density dependence of $T_{c}$ in two TEM lamellae. Figure 4(b) shows $T_{c}$ as a function of the current density changing the input current for constant geometry. One sees clearly a much weaker dependence than the one obtained changing the lamella thickness.

The behavior of the granular superconductivity at

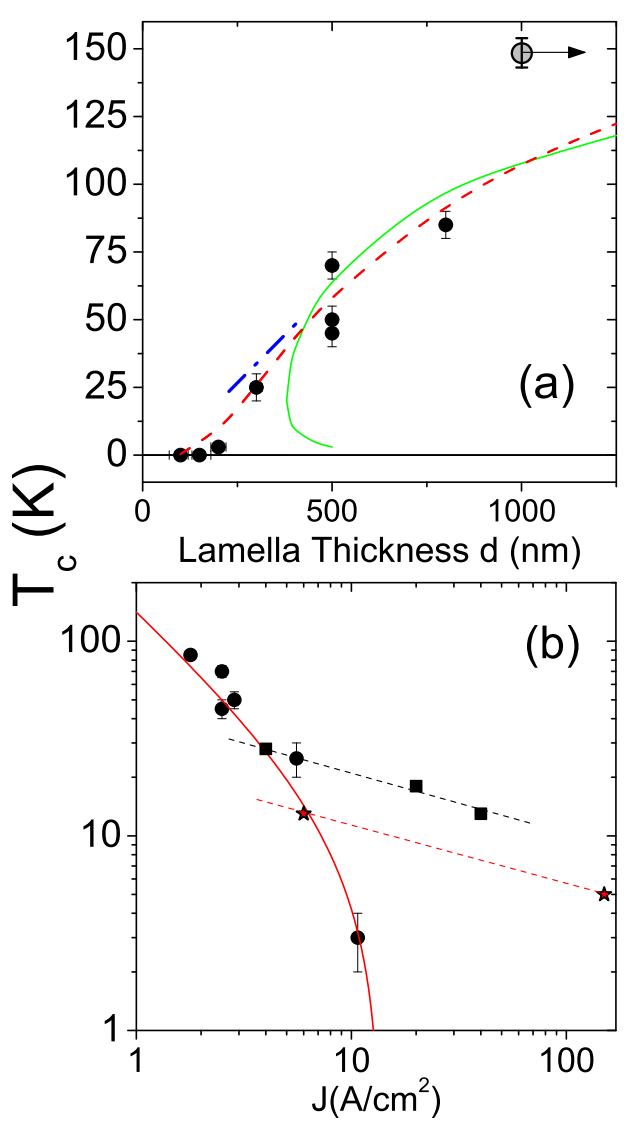

FIG. 4: (a) Critical temperature $T_{c}$ defined at the onset of the voltage decrease with decreasing temperature, obtained at $1 \mathrm{nA}$ input current vs. the thickness of the lamella. The straight dashed-dotted (blue) line represents the linear fit through the experimental points obtained for $\mathrm{Nb} / \mathrm{Al}$ multilayers from 27. (both axis must be divided by 10 in this case). The continuos (green) line follows Eq.22 proposed in [27. The dashed (red) line follows Eq. (1) with the parameters described in the text. The upper point represents the temperature of the maximum in the resistance for the bulk HOPG sample, see Fig. 2. The arrow indicates the expected range $(1 \mu \mathrm{m} \lesssim d \lesssim 10 \mu \mathrm{m})$ for the size of the interfaces in the bulk sample, taking into account TEM [20] as well as EBSD [21] measurements. (b) The critical temperature vs. the estimated current density taking into account the measured total normal area of the lamellae. The red line is a fit to the experimental values of $T_{c}$ obtained at $1 \mathrm{nA}$, which follows the function $153(1 / J)\left[\mathrm{K} \mathrm{cm}^{2} / \mathrm{A}\right]-11[\mathrm{~K}] \propto d$. The $T_{c}(J)$-curves of the two data sets $(\boldsymbol{\square}, \star$, dashed guide lines) were measured increasing the input current density in the same lamella.

graphite interfaces is certainly not as simple as in usual Josephson-coupled superconducting grains in a normal matrix. In real graphite samples we expect inhomogeneously distributed superconducting strength within the graphite interfaces due to, e.g., changes in the stacking order, differences in the doping, etc. According to 11] high-temperature superconductivity at the interfaces may survive throughout the sample due to the proxim- 
ity effect between $\mathrm{ABC} / \mathrm{ABA}$ interfaces where the order parameter is enhanced. To our knowledge there is no published theory directly applicable to understand the interface-size dependence of the critical behavior found in this work. Nevertheless, taking into account that the observed behavior can be related to the existence of superconducting and normal conducting regions, let us compare the size dependence of $T_{c}$ obtained here with the one observed in conventional superconductors. Especially interesting in this frame is the linear decrease of the superconducting critical temperature with the whole thickness of the ensemble of superconducting/metal multilayers leaving constant the thickness of each of the layers, see [27] and refs. therein. The dashed-dotted straight line in Fig. 4(a) is the experimental line obtained for $\mathrm{Nb} / \mathrm{Al}$ multilayers multiplying by 10 both axes. In this case the obtained $T_{c}(d)$ dependence of our lamellae has a nearly identical slope as that obtained for $\mathrm{Nb} / \mathrm{Al}$ multilayers, where the thickness is the total thickness $d_{T}$ [27].

The origin for the change of $T_{c}$ in conventional superconducting multilayers and thin wires has been tentatively given in [27, 28] based on weak localization (WL) corrections to $T_{c}$ for $2 \mathrm{D}$ superconductors [29]. In both, the presence of disorder affects the screening of the Coulomb interaction, and since the latter changes the BCS coupling parameter, this results into an exponential suppression of the critical temperature in ordinary BCS superconductors. However, the parameter dependence of this exponential is different in the theory [28] than the one used to fit the experimental results of $\mathrm{Nb} / \mathrm{Al}$ multilayers in [27]. Below, we compare our results to both approaches.

In 28, the important aspect of the disorder correction is screening, and therefore the relevant size scale is that of the whole conducting region, and the parameter controlling the correction is $t=\left(e^{2} /\left(2 \pi^{2} \hbar\right)\right) R_{\square}$, where $R_{\square}$ is the sheet resistance [28]. In the limit $t \ll \gamma^{2}$, where $\gamma$ is the dimensionless bare BCS coupling parameter, the effective critical temperature can then be obtained as

$$
T_{c}(d)=T_{\infty} \exp ^{-t(d) /\left(6 \gamma^{3}\right)}=T_{\infty} \exp ^{-\alpha / d}
$$

where $\alpha=t(d) /\left(6 \gamma^{3}\right)$ and $T_{\infty}$ is the bulk critical temperature. The dashed line in Fig. 4 shows a fit of this type of behavior to our data. There, we used $T_{\infty}=200 \mathrm{~K}$ and $\alpha \approx 600 \mathrm{~nm}$ yielding the resistivity $\rho \simeq 0.05 \gamma^{3} \Omega \mathrm{m}$. Note that the theory in Eq. (3) of Ref. [28, also predicts that $T_{c}(d)=0$ if $t=2 \gamma^{2}$. With our fitting parameters, this would take place for $d=200 / \gamma \mathrm{nm}$. As we observe no superconducting response any more at $d \lesssim 160 \mathrm{~nm}$, this would indicate the presence of a very large $\gamma \approx 1$, which is strictly speaking outside the validity range of the approach in [28]. For this value of $\gamma$ the estimated resistivity $\rho$ is several orders of magnitude larger than the one obtained from multilayer graphene samples without interfaces 22 .

On the other hand, 27] compares the thickness to the thermal length $\ell_{\mathrm{th}}(T)=\left(\hbar D / k_{B} T\right)^{1 / 2}$ at temperature $T$. Here, the $2 \mathrm{D}$ diffusion constant $D=v_{F} \ell / 2$, where $v_{F} \approx 10^{6} \mathrm{~m} / \mathrm{s}$ is the Fermi velocity and $\ell$ is the mean free path. Therefore, the estimated correction to the critical temperature is

$$
T_{c}(d)=T_{\infty} e^{-\ell_{\mathrm{th}}\left(T_{c}\right) / d}
$$

Independent measurements done in graphite flakes without (or with much less influence of) interfaces provide $\ell \sim 3 \mu \mathrm{m}$ at $T<100 \mathrm{~K}\left[30\right.$ ] and therefore $D \sim 1.5 \times 10^{4}$ $\mathrm{cm}^{2} / \mathrm{s}$. Note that this is four orders of magnitude larger than the one used in [27, meaning that the effect is relevant in far thicker samples or at much higher temperatures than in $\mathrm{Nb} / \mathrm{Al}$ multilayers which have $T_{c} \lesssim 10 \mathrm{~K}$. We use this diffusion constant and for $T_{\infty}=150 \mathrm{~K}$ we take the temperature of the maximum resistance measured in HOPG (see Fig. 2). The obtained numerical solution of Eq. 22 is plotted as the continuous line in Fig. 4. The semiquantitative agreement is remarkable as well as the estimated cut-off $d_{\text {min }}=\ell_{\text {th }}\left(T_{c}\right) / 2.7 \approx 0.38$ $\mu \mathrm{m}$, below which Eq. (2) has no solution.

We note that using either the theory of [28] or the model used in 27] to describe our results has many shortcomings: For interface superconductivity, we expect screening to be strongly inhomogeneous, whereas 28 assumes homogeneous superconductors and in 27] the metals did not differ very much. Moreover, even the use of the conventional weak-coupling theory in describing our results is questionable, because of the high critical temperatures. One possible explanation of this high critical temperature is associated with the flat bands emerging at the interfaces due to inclusions with rhombohedral stacking 9, 10. However, a prediction of the screening effect on such flat band superconductivity does not yet exist.

Finally, it should be noted that some evidence for the formation of charge density waves (CDW) was found in $\mathrm{CaC}_{6}$ at $\sim 250 \mathrm{~K}$, whereas its $T_{c}=11.5 \mathrm{~K}$ 31. On the other hand doping the surface of bulk graphite samples with $\mathrm{Ca}$ atoms showed hints for superconductivity at $T \sim 200 \mathrm{~K} 32$. Taking into account the available evidence on the antagonist relationship between CDW and superconductivity 33, future experiments should try to check whether CDW are also formed in HOPG interfaces and their possible relation with the size effect reported in this study.

In conclusion, we have found that the temperature at which the Josephson behavior in TEM lamellae sets in, decreases with the size of the interfaces. This behavior provides a way to understand differences in the temperature dependence of the resistance in graphite samples with interfaces. Weak localization effects appear to be a possible origin for the here reported phenomenon.

This work was partially supported by the ESF "Energie", the European Research Council (Grant No. 240362-Heattronics) and the Academy of Finland. 
[1] N. Reyren, S. Thiel, A. D. Caviglia, L. F. Kourkoutis, G. Hammerl, C. Richter, C. W. Schneider, T. Kopp, A.S. Rüetschia, D. Jaccard, et al., Science 317, 1196 (2007).

[2] A. Gozar, G. Logvenov, L. F. Kourkoutis, A. T. Bollinger, L. A. Giannuzzi, L. A. Muller, and I. Bozovic, Nature 455, 782 (2008).

[3] V. L. Ginzburg, Phys. Lett. 13, 101 (1964).

[4] D. V. Gitsu, A. F. Grozav, V. G. Kistol, L. I. Leporda, and F. M. Muntyanu, JETP Lett. 55, 403 (1992).

[5] F. M. Muntyanu and L. I. Leporda, Phys. Solid State 37, 298 (1995).

[6] F. Muntyanua, A. Gilewski, K. Nenkov, J. Warchulska, and A. Zaleski, Phys. Rev. B 73, 132507 (2006).

[7] F. M. Muntyanu, A. Gilewski, K. Nenkov, A. J. Zaleski, , and V. Chistol, Phys. Rev. B 76, 014532 (2007).

[8] F. Muntyanua, A. Gilewski, K. Nenkov, A. Zaleski, and V. Chistol, Solid State Commun. 147, 183 (2008).

[9] N. B. Kopnin, T. T. Heikkilä, and G. E. Volovik, Phys. Rev. B 83, 220503 (2011).

[10] N. B. Kopnin, M. Ijäs, A. Harju, and T. T. Heikkilä, Phys. Rev. B 87, 140503 (2013).

[11] W. A. Muñoz, L. Covaci, and F. Peeters, Phys. Rev. B 87, 134509 (2013).

[12] G. E. Volovik, J Supercond Nov Magn 26, 2887 (2013).

[13] Q. Lin, T. Li, Z. Liu, Y. Song, L. He, Z. Hu, Q. Guo, and H. Ye, Carbon 50, 2369 (2012).

[14] C. H. Lui, Z. Li, Z. Chen, P. V. Klimov, L. E. Brus, and T. F. Heinz, Nano Lett. 11, 164 (2011).

[15] K. Antonowicz, Nature 247, 358 (1974).

[16] K. Antonowicz, phys. stat. sol. (a) 28, 497 (1975).

[17] P. Esquinazi, Papers in Physics 5, 050007 (2013).

[18] I. Felner, Materials Research Express 1, 016001 (2014).

[19] M. Inagaki, New Carbons: Control of Structure and Functions (Elsevier, 2000), ISBN 0080437133.

[20] A. Ballestar, J. Barzola-Quiquia, T. Scheike, and P. Es- quinazi, New J. Phys. 15, 023024 (2013).

[21] J. C. González, M. Muñoz, N. García, J. BarzolaQuiquia, D. Spoddig, K. Schindler, and P. Esquinazi, Phys. Rev. Lett. 99, 216601 (2007).

[22] S. Dusari, J. Barzola-Quiquia, P. Esquinazi, and N. García, Phys. Rev. B 83, 125402 (2011).

[23] Y. Kawashima, AIP Advances 3, 052132 (2013).

[24] V. Ambegaokar and B. I. Halperin, Phys. Rev. Lett. 22, 1364 (1969).

[25] Y. M. Ivanchenko and L. A. Zil'berman, Zh. Eksp. Teor. Fiz. 55, 2395 (1968), (Sov. Phys. JETP 28:1272-1276, 1969). See also idem, Zh. Eksp. Teor. Fiz. Pis. Red. 8:189192 (1968) (JETP Lett. 8:113-115 (1968)).

[26] H. Kempa, Y. Kopelevich, F. Mrowka, A. Setzer, J. H. S. Torres, R. Höhne, and P. Esquinazi, Solid State Commun. 115, 539 (2000).

[27] J. Guimpel, M. E. de la Cruz, F. de la Cruz, H. J. Fink, O. Laborde, and J. C. Villegier, J Low Temp Phys 63, 151 (1986).

[28] Y. Oreg and A. Finkel'stein, Phys. Rev. Lett. 83, 191 (1999).

[29] H. Fukuyama, Physica 126B, 306 (1984), see also H. Ebisawa, H. Fukuyama, and S. Maekawa, J. Phys. Soc. Japan 54, 2257 (1985).

[30] P. Esquinazi, J. Barzola-Quiquia, S. Dusari, and N. García, J. Appl. Phys. 111, 033709 (2012).

[31] K. C. Rahnejat, C. A. Howard, N. E. Shuttleworth, S. R. Schofield, K. Iwaya, C. F. Hirjibehedin, C. Renner, G. Aeppli, and M. Ellerby, Nature Commun. 2, 558 (2011).

[32] S. W. Han, J. D. Lee, J. P. Noh, and D. W. Jung, J. Low Temp. Phys. 160, 41 (2010).

[33] A. M. Gabovich and A. I. Voitenko, Low Temperature Physics 39, 232 (2013). 